\title{
Hyperbolic conservation laws on manifolds with limited regularity
}

\author{
Philippe G. LeFloch ${ }^{a}$, Baver Okutmustur ${ }^{\text {a }}$ \\ a Laboratoire Jacques-Louis Lions, Centre National de la Recherche Scientifique \\ Université Pierre et Marie Curie (Paris 6) \\ 4 Place Jussieu, 75252 Paris, France. \\ E-mail: LeFloch@ann.jussieu.fr, Okutmustur@ann.jussieu.fr
}

\begin{abstract}
We introduce a formulation of the initial and boundary value problem for nonlinear hyperbolic conservation laws posed on a differential manifold endowed with a volume form, possibly with a boundary; in particular, this includes the important case of Lorentzian manifolds. Only limited regularity is assumed on the geometry of the manifold. For this problem, we establish the existence and uniqueness of an $L^{1}$ semi-group of weak solutions satisfying suitable entropy and boundary conditions.
\end{abstract}

\section{Résumé}

Lois de conservation hyperboliques sur les variétés à faible régularité. Nous proposons une formulation du problème de Cauchy avec conditions aux limites pour les lois de conservation hyperboliques nonlinéaires posées sur une variété différentiable munie d'une forme volume, avec ou sans bord; notre étude couvre, en particulier, le cas important des variété Lorentzienne. Nous supposons une régularité limitée sur la géometrie de la variété. Pour ce problème nous démontrons l'existence et l'unicité d'un semi-groupe $L^{1}$ de solutions faibles satisfaisant à des conditions d'entropie et à des conditions aux limites convenablement définies.

Reference of this paper : C.R. Math. Acad. Sc. Paris 346 (2008), 539--543.

Version française abrégée

Dans cette Note, nous nous intéressons aux lois de conservation hyperboliques nonlinéaires posées sur une variété. Nous proposons une formulation mathématique du problème de Cauchy lorsque la variété est seulement munie d'une forme volume. En particulier, nous pouvons poser ce problème sur une variété Lorentzienne. 
Soit $M$ une variété compacte, régulière, à $n$ dimensions, munie d'une forme volume $\omega$ de class $L^{\infty}$. Sur cette variété, considérons la loi de conservation hyperbolique nonlinéaire

$$
\partial_{t} u+\operatorname{div}_{\omega}(f(u))=0, \quad u: \mathbb{R}_{+} \times M \rightarrow \mathbb{R},
$$

où le flux $f=\left(f^{j}(\bar{u}, x)\right)$ est un champ de vecteur régulier défini sur $M$ et dépendant d'un paramètre réel $\bar{u}$. L'opérateur de divergence spatial est ici défini au sens faible des distributions. (Voir (6) dans la Version en anglais.)

Nous supposons que le flux $f$ est au plus linéaire, c'est-à-dire que pour tout champ $\alpha$ de 1-formes de classe $L^{\infty}$ définies sur $M$ il existe une constante $C_{\alpha}>0$ telle que

$$
\sup _{M}|\langle\alpha, f(\bar{u})\rangle| \leq C_{\alpha}(1+|\bar{u}|), \quad \bar{u} \in \mathbb{R} .
$$

Nous recherchons alors des solutions verifiant une condition initiale en $t=0$ :

$$
u(0, x)=u_{0}(x), \quad x \in M
$$

où $u_{0} \in L_{\omega}^{1}(M)$ est donnée. Nous disons que $f$ est compatible avec la géométrie de $M$ si

$$
\left(\operatorname{div}_{\omega} f\right)(\bar{u})=0, \quad \bar{u} \in \mathbb{R} .
$$

Nous définissons alors une notion de couple d'entropie convexe (U,F) adaptée à la variété $M$. (Voir (10) dans la Version en anglais.)

Definition 0.1 Sous les hypothèses ci-dessus, une fonction $u \in L^{\infty}\left(\mathbb{R}_{+}, L_{\omega}^{1}(M)\right)$ est appelée solution entropique du problème (1)-(3) si pour tout couple d'entropie convexe $(U, F)$ et toute fonction-test $\phi$ : $[0, \infty) \times M \rightarrow \mathbb{R}_{+}$

$$
\begin{aligned}
& \iint_{\mathbb{R}_{+} \times M}\left(U(u) \partial_{t} \phi+\langle d \phi, F(u)\rangle\right) \omega d t+\iint_{\mathbb{R}_{+} \times M}\left(\left(\operatorname{div}_{\omega} F\right)(u)-\partial_{u} U(u)\left(\operatorname{div}_{\omega} f\right)(u)\right) \phi \omega d t \\
& +\int_{M} U\left(u_{0}\right) \phi(0) \omega \geq 0 .
\end{aligned}
$$

Theorem 0.1 Soit $M$ une variété compacte sans bord et $\omega \in L^{\infty}(M)$ une forme volume uniformément définie positive sur $M$. Soit $f$ un flux compatible avec la géométrie de $M$ et satisfaisant à la condition de croissance (2). Alors, le problème (1)-(3) admet un semi-groupe contractif de solutions entropiques $u_{0} \in L_{\omega}^{1}(M) \mapsto u(t):=S_{t} u_{0} \in L_{\omega}^{1}(M)$

$$
\left\|S_{t} u_{0}-S_{t} v_{0}\right\|_{L_{\omega}^{1}(M)} \leq\left\|u_{0}-v_{0}\right\|_{L_{\omega}^{1}(M)}, \quad t \geq 0, \quad u_{0}, v_{0} \in L_{\omega}^{1}(M) .
$$

Nous étendons aussi ce résultat au cas des lois de conservation définies sur un espace-temps muni d'une métrique Lorentzienne de classe $L^{\infty}$, et incluons des conditions aux limites lorsque $M$ est une variété à bord. Lorsque la condition de compatibilité géométrique est relaxée, la distance $L^{1}$ entre deux solutions entropiques peut croître en temps. Pour plus de détails nous renvoyons le lecteur à la version en anglais et à $[9]$.

Version in English 


\section{Introduction}

In this Note we consider nonlinear hyperbolic conservation laws posed on a differentiable manifold and we introduce a formulation of the initial and boundary value problem. The manifold under consideration may have a boundary and is endowed with a Lorentzian metric or, more generally, a volume form. We introduce a notion of weak solution in the sense of distributions and formulate suitable entropy and boundary conditions. We then establish the existence and uniqueness of entropy solutions when the initial data are integrable functions and the manifold geometry has limited regularity. This problem is motivated by similar questions arising in the evolution of compressible fluids on manifolds (e.g. fluid flows on the sphere or on an Einstein spacetime of general relativity). Scalar conservation laws provide us with a simplified model for understanding certain important features arising in this context, especially the lack of regularity of the solutions and the geometry.

Our results generalize the well-posedness and convergence theory established in $[1,3,4,8,10]$ for the case of Riemannian manifolds and, earlier on, in Kruzkov's classical work [7] in the Euclidian case. Our presentation will proceed by investigating three different settings that are (related but) of independent interest in the applications: $n$-dimensional manifolds endowed with a volume form, Lorentzian manifolds, and $(n+1)$-dimensional manifolds endowed with a volume form. Our proofs use a sequence of approximate solutions constructed by the finite volume method.

\section{Conservation laws on a manifold endowed with an $L^{\infty}$ volume form}

We consider first the case that $M$ is a compact, smooth, $n$-dimensional manifold endowed with a volume form $\omega \in L^{\infty}(M)$. So, $\omega$ is a bounded Lebesgue measurable, uniformly positive $n$-form field which, in local coordinates $x=\left(x^{j}\right)_{1 \leq j \leq n}$ defined in some open set $B$, reads

$$
\omega=\bar{\omega} d x^{1} \ldots d x^{n}, \quad \bar{\omega} \in L^{\infty}(B), \quad \omega \geq \omega_{*}
$$

for some $\omega_{*}>0$ (depending on the choice of local coordinates). On this manifold, we consider the hyperbolic conservation law

$$
\partial_{t} u+\operatorname{div}_{\omega}(f(u))=0, \quad u: \mathbb{R}_{+} \times M \rightarrow \mathbb{R},
$$

in which the flux $f=\left(f^{j}(\bar{u}, x)\right)$ is a smooth vector field on $M$ depending upon a real parameter $\bar{u}$. In (5), the spatial divergence operator is defined in a weak sense from the volume form $\omega$. Namely, when $u$ and $\omega$ are smooth and the vector field $f(u)$ is supported in a local chart of coordinates, one defines

$$
\left(\operatorname{div}_{\omega} f(u)\right)(t, x):=\frac{1}{\bar{\omega}(x)} \partial_{j}\left(\bar{\omega}(x) f^{j}(u(t, x), x)\right),
$$

where implicit summation over repeated indices is used. Hence, for every smooth function $\theta: M \rightarrow \mathbb{R}$ compactly supported in a local coordinate chart and for $t \geq 0$,

$$
\begin{aligned}
\int_{M} \operatorname{div}_{\omega}(f(u))(t, \cdot) \theta \omega & =-\int_{B}\left(\partial_{j} \theta\right)(x) f^{j}(u(t, x), x) \bar{\omega}(x) d x^{1} \ldots d x^{n} \\
& =\int_{M}\langle d \theta, f(u(t, \cdot))\rangle \omega
\end{aligned}
$$

where the one-form field $d \theta$ is the differential of the function $\theta$ and $\langle\cdot, \cdot\rangle$ denotes the bracket between forms and vectors on $M$. The above formula allows one to reformulate the conservation law (5) in a weak 
sense when $\omega \in L^{\infty}(M), u \in L_{\omega}^{1}(M)$, and the flux $f$ is at most linear, i.e., for every $L^{\infty}$ field $\alpha$ of 1 -forms on $M$ there exists a constant $C_{\alpha}>0$ such that

$$
\sup _{M}|\langle\alpha, f(\bar{u})\rangle| \leq C_{\alpha}(1+|\bar{u}|), \quad \bar{u} \in \mathbb{R} .
$$

We are interested in solutions satisfying the following initial condition at the time $t=0$ :

$$
u(0, x)=u_{0}(x), \quad x \in M,
$$

where the function $u_{0} \in L_{\omega}^{1}(M)$ is given. Generalizing [3], we say that $f$ is geometry-compatible if

$$
\left(\operatorname{div}_{\omega} f\right)(\bar{u})=0, \quad \bar{u} \in \mathbb{R} .
$$

By definition, a Lipschitz continuous function $U: \mathbb{R} \rightarrow \mathbb{R}$ together with a family of vector fields $F=F(\bar{u})$ depending Lipschitz continuously upon the parameter $\bar{u}$ is called an entropy pair for the conservation law (5) if for almost all $\bar{u} \in \mathbb{R}$

$$
\partial_{u} F(\bar{u})=\partial_{u} U(\bar{u}) \partial_{u} f(\bar{u}) .
$$

Finally, we introduce the notion of entropy solution.

Definition 2.1 Under the above assumptions, a function $u \in L^{\infty}\left(\mathbb{R}_{+}, L_{\omega}^{1}(M)\right)$ is called an entropy solution to the initial value problem (5)-(8) if for every entropy pair $(U, F)$ and every smooth function $\phi \geq 0$ with compact support in $[0, \infty) \times M$,

$$
\begin{aligned}
& \iint_{\mathbb{R}_{+} \times M}\left(U(u) \partial_{t} \phi+\langle d \phi, F(u)\rangle\right) \omega d t+\iint_{\mathbb{R}_{+} \times M}\left(\left(\operatorname{div}_{\omega} F\right)(u)-\partial_{u} U(u)\left(\operatorname{div}_{\omega} f\right)(u)\right) \phi \omega d t \\
& +\int_{M} U\left(u_{0}\right) \phi(0) \omega \geq 0 .
\end{aligned}
$$

Theorem 2.1 Let $M$ be a compact, smooth manifold without boundary and $\omega \in L^{\infty}(M)$ be a uniformly positive volume form on $M$. Let $f$ be a geometry-compatible flux on $M$ satisfying the growth condition (7). Then, the initial value problem (5)-(8), admits a contractive semi-group of entropy solutions $u_{0} \in$ $L_{\omega}^{1}(M) \mapsto u(t):=S_{t} u_{0} \in L_{\omega}^{1}(M)$,

$$
\left\|S_{t} u_{0}-S_{t} v_{0}\right\|_{L_{\omega}^{1}(M)} \leq\left\|u_{0}-v_{0}\right\|_{L_{\omega}^{1}(M)}, \quad t \geq 0, \quad u_{0}, v_{0} \in L_{\omega}^{1}(M) .
$$

The above definition and theorem extend a classical result by Kruzkov [7], which was restricted to equations posed on the (flat) Euclidian space. The proof follows the lines of arguments in [3] and relies on the observation that the volume form structure $\omega$ only is required to carry out all of the arguments therein. Indeed, the metric can be eliminated and replaced by the corresponding expressions in terms of the volume form. Note also that boundary conditions can also be included if $M$ has a boundary and the condition (9) can also be relaxed; see [9] for further details.

\section{Conservation laws on a spacetime endowed with a Lorentzian metric}

We now discuss a setting where further geometric structure is provided on the manifold; this is motivated by problems arising in the theory of general relativity. For simplicity in the presentation, we assume in 
this section that the geometry is sufficiently smooth, and we refer the following section for the case of a limited regularity. We assume that a smooth, $(n+1)$-dimensional manifold $M$ with smooth boundary $\partial M$ is endowed with a smooth Lorentzian metric $g$ with signature $(-,+, \ldots,+)$. We denote by $d v_{M}$ the volume form on $(M, g)$. We say, in short, that $(M, g)$ is a spacetime.

A flux on the manifold $M$ is still defined as a smooth vector field $f=f(\bar{u}, x)$ depending on a parameter $\bar{u}$, but it is important to observe that time and space, now, are handled together. So, in local coordinates we may write $x=\left(x^{\alpha}\right)=\left(t, x^{j}\right)$, with $\alpha=0, \ldots, n$ and $j=1, \ldots, n$, and we set $f=\left(f^{j}(\bar{u}, x)\right)$. We consider the following conservation law posed on $M$ :

$$
\operatorname{div}_{g}(f(u))=0, \quad u: M \rightarrow \mathbb{R} .
$$

To formulate the initial and boundary value problem associated with (11), we prescribe a measurable and bounded function $u_{B}: \partial M \rightarrow \mathbb{R}$ defined on the boundary $\partial M$ of the spacetime, and we search for a function $u \in L^{\infty}(M)$ satisfying (11) in the distributional sense, together with an entropy condition, such that the (weak) trace of $u$ on $\partial M$ satisfies the boundary condition

$$
\left.u\right|_{\partial M} \in \mathcal{E}_{N}\left(u_{0}\right)
$$

in a sense specified now. By definition, a convex function $U: \mathbb{R} \rightarrow \mathbb{R}$ and a vector field $F=F(\bar{u})$ is called a convex entropy pair associated with the conservation law (11) if, in local coordinates,

$$
F^{j}(\bar{u}, x)=\int_{0}^{\bar{u}} \partial_{u} U\left(u^{\prime}\right) \partial_{u} f^{j}\left(u^{\prime}, x\right) d u^{\prime}, \quad \bar{u} \in \mathbb{R} .
$$

Here, $N$ denote the field of unit normal 1-forms along the boundary, and for all convex entropy pair $(U, F)$ we set

$$
\mathcal{E}_{N}\left(u_{0}\right):=\left\{\bar{u} \mid E_{N}\left(u_{B}, \bar{u}\right):=\left\langle N, F\left(u_{B}\right)\right\rangle+\partial_{u} U\left(u_{B}\right)\left\langle N, f(\bar{u})-f\left(u_{B}\right)\right\rangle \leq\langle N, F(\bar{u})\rangle\right\} .
$$

In the boundary condition (12) we do not distinguish between space-like parts (initial hypersurface, final hypersurface) or time-like parts of the boundary $\partial M$. If $S \subset \partial M$ is a space-like hypersurface then the boundary condition reduces to either a vacuous requirement or else to the continuity property $\left.u\right|_{S}=u{ }_{B}$.

Definition 3.1 A Young measure $\nu: M \rightarrow \operatorname{Prob}(\mathbb{R})$ with compact range is called an entropy measurevalued solution to the problem (11)-(12) if for some scalar field $b \in L^{\infty}(\partial M)$ the inequalities

$$
\int_{M} \prec \nu,\langle d \theta, F\rangle+\left(d i v_{g} F-\partial_{u} U\left(d i v_{g} f\right)\right) \theta \succ d v_{M}+\int_{\partial M} \prec \nu, E_{N}\left(u_{B}, b\right) \succ \theta d v_{\partial M} \geq 0
$$

hold for all convex entropy pairs $(U, F)$ and all smooth functions $\theta \geq 0$ compactly supported in $\bar{M}$. On the other hand, a function $u \in L^{\infty}(M)$ is called an entropy solution to the problem (11)-(12) if and only if the associated Young measure $\delta_{u}$ (the Dirac measure at the point $u$ ) is an entropy measure-valued solution to the same problem.

The above definition extends a notion introduced by DiPerna [5] for equations posed in the (flat) Euclidian space and by Szepessy [11] and Kondo and LeFloch [6] for the problem with boundary conditions. The formulation and the convergence of finite volume schemes on a Lorentzian manifold without boundary was treated in [2]. In the following theorem we suppose that $M$ is foliated by oriented space-like hypersurfaces, that is, $M=\bigcup_{t \geq 0} H_{t}$. 
Theorem 3.1 Suppose that $(M, g)$ is a time-oriented, smooth, $(n+1)$-dimensional Lorentzian manifold foliated by oriented space-like hypersurfaces $H_{t}$. Let $f=f(\bar{u})$ be a future-oriented, time-like, smooth vector field depending on a parameter. Then, for each data $u_{B} \in L^{\infty}(\partial M) \cap L_{g}^{1}(\partial M)$, the initial and boundary value problem (11)-(12) admits a unique entropy solution $u=S_{t} u_{B} \in L^{\infty}(M)$ such that $u_{H_{t}} \in L_{g}^{1}\left(H_{t}\right)$ for all times $t \geq 0$, with moreover for each $T>0$ and $t \in[0, T]$

$$
\left\|S_{T} u_{0}-S_{T} v_{0}\right\|_{L_{g}^{1}\left(H_{t}\right)} \leq C_{T}\left\|u_{B}-v_{B}\right\|_{L_{g}^{1}\left((\partial M)_{T}\right)}, \quad u_{B}, v_{B} \in L_{g}^{1}(\partial M) \cap L^{\infty}(\partial M),
$$

where $(\partial M)_{T}$ is the part of the boundary corresponding to $\{0 \leq t \leq T\}$ and the constant $C_{T}$ depends on $T$ and the sup norm of the data, only.

We can show that, in the integral term $\left\|u_{B}-v_{B}\right\|_{L_{g}^{1}\left((\partial M)_{T}\right)}$, one can suppress the part along which the vector field $\partial_{u} f(\bar{u}, \cdot)$ is outgoing for all $\bar{u}$.

\section{Conservation laws on a spacetime endowed with an $L^{\infty}$ volume form}

Finally, the above results are extended to the situation where $M$ is an $(n+1)$-dimensional spacetime endowed with a volume form $\omega$ (rather than a Lorentzian metric) and, moreover, is solely of class $L^{\infty}$. Given a parameter-dependent vector field $f$ with "at most linear" growth, weak solutions in $L_{\text {loc }}^{1}(M)$ to the hyperbolic conservation law

$$
\operatorname{div}_{\omega}(f(u))=0, \quad u: M \rightarrow \mathbb{R}
$$

are defined by adapting the definition introduced in Section 2. Observe that the (spacetime) volume form need not admit a (strong) trace on $n$-dimensional hypersurfaces in $M$, so that the conclusions of Theorems 2.1 and 3.1 hold in a weak sense only. We refer to [9] for further details.

\section{References}

[1] P. Amorim, M. Ben-Artzi, and P.G. LeFloch, Hyperbolic conservation laws on manifolds: total variation estimates and the finite volume method, Meth. Appl. Anal. 12 (2005), 291-324.

[2] P. Amorim, P.G. LeFloch, and B. Okutmustur, Finite volume schemes on Lorentzian manifolds, preprint, arXiv:0712.122.

[3] M. Ben-Artzi, and P.G. LeFloch, The well-posedness theory for geometry compatible hyperbolic conservation laws on manifolds, Ann. Inst. H. Poincaré : Nonlin. Anal. 24 (2007), 989-1008.

[4] M. Ben-Artzi, J. Falcovitz, and P.G. LeFloch, Hyperbolic conservation laws on the sphere. A geometry compatible finite volume scheme, preprint, arXiv:0808.2062

[5] R.J. DiPerna, Measure-valued solutions to conservation laws, Arch. Rational Mech. Anal. 88 (1985), 223-270.

[6] C. Kondo and P.G. LeFloch, Measure-valued solutions and well-posedness of multi-dimensional conservation laws in a bounded domain, Portugal. Math. 58 (2001), 171-194.

[7] S.N. Kruzkov, First-order quasilinear equations with several space variables, Math. USSR Sb. 10 (1970), $217-243$.

[8] P.G. LeFloch, Hyperbolic conservation laws and spacetimes with limited regularity, Proc. Inter. Conf. on Hyper. Problems: theory, numerics, and application, July 2006, Lyon, France.

[9] P.G. LeFloch and B. Okutmustur, in preparation.

[10] E.Y. Panov, On the Cauchy problem for a first-order quasilinear equation on a manifold, Differential Equations 33 (1997), 257-266. 
[11] A. Szepessy, Measure-valued solutions of scalar conservation laws with boundary conditions, Arch. Rational Mech. Anal. 107 (1989), 181-193. 